\title{
Prevalencia de la incontinencia fecal en centros de salud y casas de reposo
}

\author{
Alejandro J Zárate ${ }^{1}$, Francisco López-Köstner ${ }^{2,4}$, \\ Flavia Vergara ${ }^{a}$, Nathalia Badillaa, Paola Viviani ${ }^{3}$. \\ Prevalence of fecal incontinence \\ in health centers \\ and nursing home residents
}

Background: Fecal and urinary incontinence (FI and UI) are psychologically and socially debilitating and embarrassing conditions. Aim: To determine the prevalence of FI and associated factors in patients assessed in health centers (HC) and nursing home $(\mathrm{NH})$ residents. Material and Methods: Prospective, multicentric survey carried out in 3 $\mathrm{HC}$ and $16 \mathrm{NH}$ of Santiago. Patients and residents of NH were interrogated about FI between March and July, 2004. Results: The questionnaire was answered by 618 patients attending health centers aged 18 to 87 years (75\% females) and 128 nursing home residents aged 40 to 103 years (80\% females). The prevalence of liquid or solid FI in $\mathrm{HC}$ and $\mathrm{NH}$ was 2.7 and $45 \%$, respectively. Among the latter, the incontinence for liquids and solids was $19 \%$ and $42 \%$, respectively. The prevalence of UI was 30.1 and $62.7 \%$ in $\mathrm{HC}$ patients and $\mathrm{NH}$ residents, respectively. Among patients attending $\mathrm{HC}$, multivariable analysis showed a relation between older age and urinary incontinence with liquid or solid FI. Among NH residents, multivariate analysis showed a relation between urinary incontinence and motor disabilities with liquid or solid FI. Conclusions: The prevalence of liquid or solid FI among patients assessed in health center is 2.7\%. This figure increases in older people, those with UI and in NH residents (Rev Méd Chile 2008; 136: 867-72).

(Key w ords: Fecal incontinence; Health centers, ambulatory; Nursing homes)

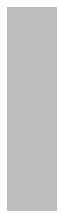

Recibido el 29 de agosto, 2007. Aceptado el 10 de marzo, 2008.

${ }^{1}$ Programa de Formación como especialista en Cirugía General, Pontificia Universidad Católica de Chile. ${ }^{2}$ Departamento de Cirugía Digestiva, Pontificia Universidad Católica de Chile. ${ }^{3}$ Departamento de Salud Pública, Pontificia Universidad Católica de Chile. Santiago de Chile. ${ }^{4}$ Unidad de Coloproctología, Clínica Las Condes.

aAlumna de Escuela de Medicina, Facultad de Medicina, Pontificia Universidad Católica de Chile.

$\mathrm{S}_{\mathrm{p}}^{\mathrm{e}}$ define como incontinencia fecal (IF) la pérdida de deposiciones en forma involuntaria en un momento o lugar socialmente inapropiado ${ }^{1}$. Esta patología junto a la incontinencia de orina

Correspondencia a: Dr. Francisco López Köstner. Unidad de Coloproctología, Clínica Las Condes. Lo Fontecilla 441, Las Condes, Santiago. Teléfono: 4888101. Fax: 4888129.

E mail: flopez@clinicalascondes.cl
(IU), pueden ser una de las condiciones sicológica y socialmente más debilitantes de un individuo. Pueden predisponer a aislamiento social, pérdida de la autoestima, depresión, además de condicionar elevados costos directos e indirectos ${ }^{2,3}$, así como también, presentar mayor cantidad de enfermedades asociadas a estas condiciones ${ }^{4}$. En el ámbito de la geriatría, la IF es considerada como una dolencia frecuente. En estudios realizados en 
Estados Unidos de Norteamérica (EE.UU.) la IF se encuentra entre las primeras 5 patologías más frecuentes de admisión a casas de reposo. Hasta hace una década, tanto la difusión del problema de la incontinencia, como la posibilidad de estudio diagnóstico y tratamiento satisfactorio, era limitada, esto implicaba que los pacientes afectados tenían pocas posibilidades de mejoría sintomática.

El estudio de la IF en la población general demuestra una prevalencia entre $1 \%$ y $7 \% 5$, la cual aumenta al ser estudiada en la población que acude a un centro de atención de salud por otro motivo ${ }^{6}$. Al considerar a los mayores de 65 años esta cifra se eleva hasta 15\%. Las cifras de prevalencia correspondientes a los residentes en casas de reposo son aún mayores, correspondiendo a cifras que bordean $50 \%{ }^{3}$. Estas cifras pueden no ser exactas, ya que en general, se subestiman, debido en parte al no relato de los síntomas de incontinencia al personal de salud por parte de los pacientes afectados ${ }^{7}$. Considerando a Chile como un país con una población que tiende al envejecimiento, consideramos relevante estudiar la prevalencia de esta patología en nuestro medio. El objetivo del estudio es determinar la prevalencia de incontinencia fecal y factores asociados en personas que acuden a un centro de salud (CDS) $\mathrm{y}$ en residentes de casas de reposo (CDR).

\section{MATERIALES Y MÉTODO}

Se diseñó un estudio prospectivo en dos tipos de poblaciones. En pacientes que acuden por cualquier motivo a un CDS y en personas que residen en CDR, el estudio se realizó entre marzo y julio de 2004, mediante encuestas. La encuesta comprendió la identificación de los pacientes, factores de riesgo para presentar incontinencia fecal, identificación del tipo de incontinencia y su severidad, esto último mediante la graduación de las tres primeras preguntas del score de $W_{e x n e r}^{8}$, es decir, la frecuencia de la incontinencia a gases, deposiciones líquidas y sólidas. Por último, se preguntó acerca de la comunicación de sus síntomas a terceras personas. Además, en pacientes mujeres que acuden a un CDS se preguntó por factores de riesgo ginecológicos, que pueden asociarse a IF.
La presencia de IF se definió como el escape de gases, deposiciones líquidas o sólidas de forma involuntaria por el ano, con al menos un evento por semana. La IU se definió para nuestros propósitos, como el escape de orina involuntario de al menos una vez por semana.

Se encuestó a pacientes de 3 centros de salud y personas residentes en 16 casas de reposo de Santiago, todas las personas encuestadas eran mayores de 18 años. En todos los centros en donde se llevó a cabo el estudio, se obtuvo el consentimiento tanto de parte de los médicos o directores del centro, como de los pacientes.

El tamaño de la muestra se estimó en 156 y 470 personas para obtener la prevalencia de la IF en residentes de CDR y de CDS de Santiago, respectivamente. Para el cálculo se consideró una prevalencia estimada de IF de $30 \%$ y una variación de $10 \%$ en CDR, y de $20 \%$ con una variación de $5 \%$ para CDS. En ambos casos se consideró una potencia de $80 \%$ y nivel de confianza de $95 \%$. Se realizó corrección por finitud, considerando 845 casos totales en CDR en Santiago.

Las encuestas fueron realizadas por personal de salud y alumnos de medicina y fueron ingresadas prospectivamente a una base de datos computacional. Las encuestas en los CDS se realizaron en días hábiles a pacientes atendidos cuyo motivo principal de consulta no fuese IF.

El análisis estadístico se realizó mediante los test de chi cuadrado, $\mathrm{T}$ de Student y regresión logística binaria. Consideramos estadísticamente significativo un valor $\mathrm{p}<0,05$.

\section{Resultados}

En el periodo descrito se obtuvo un total de 618 y 161 encuestas completadas en CDS y CDR respectivamente, las que fueron incluidas para ser analizadas. No hubo problemas de disposición a responder por parte de los encuestados. En CDS correspondieron al sexo femenino 462 pacientes $(74,7 \%)$ y la edad promedio fue de 47,4 años (i: 18-87). En CDR, 128 pacientes (79,5\%) correspondieron al sexo femenino y la edad promedio fue de 84,1 años (i: 40-103) (Tabla 1).

En pacientes que acuden a CDS se observó una prevalencia de IF de líquidos, sólidos 0 
Tabla 1. D escripción general de personas en centros de salud y casas de reposo

\begin{tabular}{|lrrrl|}
\hline Variable & \multicolumn{2}{l|}{ CDS } & \multicolumn{2}{l|}{ CDR } \\
\hline Total personas & 618 & 161 & \\
Edad promedio en años & 47,4 & 84,1 & \\
Sexo femenino (\%) & 462 & $(75)$ & 128 & $(79,5)$ \\
Mujer con IF a gases (\%) & 110 & $(23,9)$ & $\mathrm{NE}$ & \\
Mujeres con IF a líquidos (\%) & 12 & $(2,6)$ & 29 & $(22,6)$ \\
Mujeres con IF a sólidos (\%) & 7 & $(1,5)$ & 58 & $(45,3)$ \\
Mujeres con IU (\%) & 176 & $(38,1)$ & 84 & $(65,6)$ \\
Hombres con IF a gases (\%) & 17 & $(10,9)$ & NE & \\
Hombres con IF a líquidos (\%) & 2 & $(1,2)$ & 2 & $(6,0)$ \\
Hombres con IF a sólidos (\%) & 1 & $(0,6)$ & 10 & $(30,3)$ \\
Hombres con IU (\%) & 10 & $(6,4)$ & 17 & $(54,5)$ \\
\hline
\end{tabular}

Nota: CDS: Centro de salud. CDR: casas de reposo. IF: Incontinencia fecal. IU: Incontinencia urinaria. NE: No evaluado.

ambos en 2,7\% (2,2\% y 1,3\% a líquidos y sólidos, respectivamente). Además, se encontró una prevalencia para IF a gases de $20,5 \%$.

$\mathrm{Al}$ analizar uni y multivariadamente solamente a las personas con IF a líquidos o sólidos (17 personas) se obtiene un mayor riesgo sólo para la mayor edad (OR: 1,04; IC: 1,01-1,08, $p=0,021$ ) y la presencia de incontinencia urinaria (OR: 6,6; IC: 2,1-20,7, $\mathrm{p}=0,001$ ).

En el análisis univariado de los pacientes con IF a gases, la presencia de IF a gases se relacionó con: mayor edad (con IF 53,5 años vs sin IF 45,9 años, $\mathrm{p}=0,001$ ), sexo femenino (mujeres con IF $23,8 \%$ vs hombres con IF 10,9\%, p $=0,001$ ), antecedente de cirugía en la zona perineal (con cirugía, con IF 53,1\% vs sin cirugía, con IF 18,7\% p $<0,0001$ ) e incontinencia de orina (con IU, con IF $31,1 \%$ vs sin IU, con IF $15,9 \%, p<0,0001)$. El análisis multivariado de factores para IF a gases demostró los mismos factores que el análisis univariado, a excepción de la incontinencia de orina (Tabla 2).

Por otra parte, la IU presentó una prevalencia en CDS de $30,1 \%$, siendo en hombres $6,4 \%$ y en mujeres $38,1 \%$.

$\mathrm{Al}$ analizar sólo el grupo de mujeres en CDS ( $\mathrm{n}=462$ ), el análisis univariado demostró asociación con IF de cualquier tipo $(n=115)$ con mayor edad, paridad, antecedentes de cirugía perineal e incontinencia de orina. El análisis multivariado sólo demostró asociación estadísticamente significativa con la mayor edad (OR: 1,03; IC: 1,01-1,04, $\mathrm{p}<0,0001$ ) y antecedente de cirugía perineal (OR: 2,91; IC: 1,3-6,53, $\mathrm{p}=0,01$ ).

Al analizar sólo el grupo de mujeres de CDS que habían tenido hijos $(\mathrm{n}=418)$ se observó luego

Tabla 2. Análisis multivariado de factores de riesgo para poseer incontinencia fecal a gases en centros de salud

\begin{tabular}{|lccr|}
\hline Variable & Odds ratio & IC 95\% & Valor P \\
\hline Edad (por año adicional) & 1,03 & $1,02-1,04$ & $<0,001$ \\
Sexo femenino & 1,85 & $1,02-3,3$ & 0,042 \\
Antecedente de cirugía perineal & 3,07 & $1,42-6,61$ & 0,004 \\
Incontinencia de orina & 1,53 & $0,98-2,38$ & 0,062 \\
\hline
\end{tabular}

Nota: IC: Intervalo de confianza. 
del análisis multivariado para IF a líquidos y sólidos (14 mujeres en conjunto), una asociación con mayor edad e incontinencia de orina. Además, una tendencia a presentar incontinencia si es que había tenido hijos mayores de $4.000 \mathrm{~g}$ (Tabla 3).

En personas residentes en CDR, se observó una prevalencia asociada de IF a deposiciones líquidas o sólidas de 44,7\%. La prevalencia específica de IF a líquidos y sólidos fue 19,2\% y 42,2\%, respectivamente.

En el análisis univariado, los factores que mostraron asociación con IF a deposiciones líquidas o sólidas en residentes CDR fueron: mayor edad (con IF 86,4 años vs sin IF 82,3 años, $p$ $=0,003$ ), dependencia para movilizarse (dependiente con IF $63 \%$ vs independiente con IF $14,7 \%$, $\mathrm{p}<0,0001$ ) e incontinencia de orina (con IU 67,3\% vs sin IU 6,6\%, p <0,0001). Los factores que resultaron sin asociación estadísticamente significativa para poseer IF a deposiciones líquidas o sólidas en CDR fueron: sexo femenino (mujeres con IF $48,4 \%$ vs hombres con IF $30,3 \%$, p =0,062) y tiempo desde el ingreso a la CDR (con IF 42,8 meses vs sin IF 32,3 meses, $\mathrm{p}=0,52$ ). El análisis multivariado demostró asociación de la IF a deposiciones líquidas o sólidas en residentes de
CDR con dependencia para movilizarse e incontinencia de orina (Tabla 4).

La incontinencia urinaria alcanzó una prevalencia general en CDR de 62,7\%, variando de $51,5 \%$ en hombres a $65,6 \%$ en mujeres.

Al consultar a los pacientes de CDS acerca de la comunicación de sus síntomas a otras personas, solamente $41,1 \%$ de los pacientes con IF líquida 0 sólida los habían comentado a terceras personas, y aún menos (23,5\%) a alguien del personal de salud.

\section{DisCUSIÓN}

La incontinencia fecal en la actualidad se está reconociendo como uno de los problemas emergentes en poblaciones ancianas. En décadas pasadas era poco difundido, en parte por la falta de reconocimiento de factores de riesgo en los diferentes pacientes, como por las escasas alternativas terapéuticas con que se contaba. En Chile se está observando un progresivo envejecimiento de la población general, motivo por el cual este tipo de patologías se irá haciendo cada vez más frecuente, constituyendo probablemente un problema de salud a diferentes escalas de atención.

\section{Tabla 3. Análisis multivariado de factores de riesgo para poseer incontinencia fecal a líquidos y sólidos en mujeres con hijos en centros de salud}

\begin{tabular}{|lccc|}
\hline Variable & Odds ratio & IC 95\% & Valor P \\
\hline Edad (por año adicional) & 1,05 & $1,02-1,1$ & 0,01 \\
Incontinencia urinaria & 5,3 & $1,44-20,03$ & 0,01 \\
Hijo mayor de 4.000 g & 2,88 & $0,93-8,91$ & 0,06 \\
\hline
\end{tabular}

Nota: IC: Intervalo de confianza.

Tabla 4. Análisis multivariado de factores de riesgo para poseer incontinencia fecal a deposiciones líquidas o sólidas en residentes de casas de reposo

\begin{tabular}{|lccc|}
\hline Variable & Odds ratio & IC 95\% & Valor P \\
\hline Dependencia para movilizarse & 6,1 & $2,4-15,6$ & $<0,0001$ \\
Incontinencia urinaria & 20,7 & $6,6-64,3$ & $<0,0001$ \\
\hline
\end{tabular}

Nota: IC: Intervalo de confianza. 
El análisis de las cifras obtenidas en personas que acuden a CDS demuestra que la prevalencia de IF a gases es un problema frecuente en este tipo de población. Además la IF a líquidos y sólidos en conjunto representan $2,7 \%$ de las personas que acuden a un servicio de salud por otro motivo, lo cual constituye un problema, porque menos de la mitad de las personas afectadas comenta su problema a terceros y aun en menor medida lo comenta al personal de salud. La baja manifestación de síntomas referidos a médicos, asociado a una baja sospecha, podrían favorecer una falta de diagnóstico de esta patología, ya descrito en estudios previos 7,9 .

Uno de los factores asociados a IF a gases sería la realización de algunas cirugías proctológicas $^{10,11}$, variando la tasa de incontinencia fecal posterior a la cirugía hasta cifras del orden de $50 \%$. Este factor en nuestra serie sólo se asoció a la IF a gases en personas que acudieron a CDS.

En publicaciones internacionales se describe el sexo femenino como uno de los factores involucrados en el mayor riesgo de poseer incontinencia fecal ${ }^{12,13}$. En el presente estudio encontramos un riesgo aumentado de poseer IF a gases en mujeres respecto a los hombres que acuden a CDS. Este mayor riesgo no es encontrado en todos los estudios, ya que algunos no han encontrado diferencias significativas de prevalencia de IF al comparar según el sexo de la persona ${ }^{5,14}$. A diferencia de lo encontrado en CDS, en los residentes de CDR no encontramos que las mujeres tuviesen un mayor riesgo de IF (líquida 0 sólida).

Se han reportado como factores para un incremento de la prevalencia de IF en mujeres, el haber tenido hijos, en especial si éstos se tuvieron vía vaginal. Lo anterior ya que el trauma obstétrico puede ocasionar daño a los nervios de la zona ${ }^{15}$, así como también disrupción del esfínter luego del parto ${ }^{16-21}$. En el caso de mujeres que acudían a CDS, observamos que el haber tenido hijos se relacionó significativamente con la IF de cualquier tipo en el análisis univariado. Sin embargo, al analizar todos los factores estudiados mediante un análisis multivariado, el haber tenido hijos no alcanzó a tener significancia estadística. En el análisis de mujeres que residían en CDR, el haber tenido hijos no se correlacionó con la presencia de incontinencia fecal. Así, el tener hijos es un punto a considerar para la génesis de la incontinencia, sin embargo, este dato hay que complementarlo con información adicional como el peso del recién nacido, tipo de parto, uso de fórceps y eventuales desgarros y formas de resolución del mismo $^{22}$.

Respecto de la prevalencia de incontinencia fecal en las CDR, lo publicado en la literatura varía entre $46 \%$ y $55 \% 3,23$. En nuestra serie, la IF de cualquier tipo en CDR se encuentra en el límite inferior de lo mencionado $(44,7 \%)$, esta cifra se diferencia según sexo, con una tendencia a ser mayor en mujeres que en hombres ( $p=0,06$ en análisis univariado). Si bien, el ser mujer también aparece con una tendencia a presentar mayor IF en el grupo de personas que residen en CDR, en estas personas aparecen otros factores y comorbilidades que afectan la presencia de IF. En este sentido, en las mujeres de CDR, el haber tenido hijos no confiere en nuestro grupo de estudio mayor riesgo de IF.

$\mathrm{Al}$ analizar otros factores para IF en residentes de CDR, evidenciamos que la mayor edad, la dependencia para movilizarse y la incontinencia urinaria resultan ser los factores asociados a IF. Así, observamos que en estos pacientes las posibles causas de incontinencia son diferentes a las observadas en personas que acuden a CDS, destacando que la poca autovalencia y la presencia de incontinencia urinaria son los factores mayormente asociados en nuestro estudio. Estos factores, así como el deterioro mental, han sido enunciados en estudios previos como asociados a $\mathrm{IF}^{24,25}$.

La prevalencia de IU se encuentra en el rango descrito en otras publicaciones $21,26,27$, siendo mayores a los de IF. También se ha descrito esta diferencia al comparar a personas de la misma edad según su lugar de residencia, siendo mayor en los que residen en $\mathrm{CDR}^{9}$.

En resumen, en CDS la prevalencia de IF a deposiciones líquidas o sólidas es superior a $1 \%$, asociándose principalmente a una mayor edad e incontinencia de orina. En CDR la prevalencia es mayor que en CDS, asociándose principalmente a falta de autovalencia e incontinencia de orina. 


\section{REFERENCIAS}

1. Lamah M, Kumar D. Fecal incontinence. Dig Dis Sci 1999; 44: 2488-99.

2. Meligren A, Jensen LL, Zetterstrom JP, Wong WD, HOFmeister JH, Lowry AC. Long-term cost of fecal incontinence secondary to obstetric injuries. Dis Colon Rectum 1999; 42: 857-65.

3. BoRRIE MJ, DAVIDSON HA. Incontinence in institutions: costs and contributing factors. CMAJ 1992; 147: 322-8.

4. Alessi CA, Ouslander JG, Maldague S, Al-Samarrai NR, Saliba D, Osterweil D et al. Incidence and costs of acute medical conditions in long-stay incontinent nursing home residents. J Am Med Dir Assoc 2002; 3: 229-42.

5. Nakanishi $N$, Tatara $K$, Naramura $H$, Fujiwara $H$, TAKASHIMA Y, FUKUDA H. Urinary and fecal incontinence in a community-residing older population in Japan. J Am Geriatr Soc 1997; 45: 215-9.

6. BANo F, BarRington JW. Prevalence of anorectal dysfunction in women attending health care services. Int Urogynecol J Pelvic Floor Dysfunct 2007; 18: 57-60.

7. Leigh RJ, Turnberg LA. Faecal incontinence: the unvoiced symptom. Lancet 1982; 1: 1349-51.

8. JoRGE JM, WEXNER SD. Etiology and management of fecal incontinence. Dis Colon Rectum 1993; 36: 77-97.

9. EDWARDS NI, Jones D. The prevalence of fecal incontinence in older people living at home. Age Ageing 2001; 30: 503-7.

10. Pernikoff BJ, Eisenstat TE, Rubin RJ, Oliver GC, Salvati EP. Reappraisal of partial lateral internal sphincterotomy. Dis Colon Rectum 1994; 37: 1291-5.

11. Del Pino A, Nelson RL, Pearl RK, Abcarian H. Island flap anoplasty for treatment of transsphincteric fistula-in-ano. Dis Colon Rectum 1996; 39: 224-6.

12. CAMPBEll AJ, ReinKEn J, Mccosh L. Incontinence in the elderly: prevalence and prognosis. Age Ageing 1985; 14: $65-70$.

13. Helistrom L, Ekelund P, Milsom I, Skoog I. The influence of dementia on the prevalence of urinary and faecal incontinence in 85-year-old men and women. Arch Gerontol Geriatr 1994; 19: 11-20.

14. TAley NJ, O’KeEfe EA, Zinsmeister AR, Melton LJ $3^{\text {RD }}$. Prevalence of gastrointestinal symptoms in the elderly: a population-based study. Gastroenterology 1992; 102: 895-901.
15. Snooks SJ, Setchell M, Swash M, Henry MM. Injury to the innervation of the pelvic floor sphincter musculature in childbirth. Lancet 1984; 2: 546-50.

16. Tetzschner T, Sorensen M, Lose G, Christiansen J. Anal and urinary incontinence in women with obstetric anal sphincter rupture. Br J Obstet Gynaecol 1996; 103: 1034-40.

17. Sultan AH, Kamm MA, Hudson CN, Thomas JM, BARTRAM CI. Anal-sphincter disruption during vaginal delivery. N Engl J Med 1993; 329: 1905-11.

18. Donnelly V, Fynes M, CAMPBell D, Johnson $H$, O'CONNELL PR, O'HeRLHHY C. Obstetric events leading to anal sphincter damage. Obstet Gynecol 1998; 92: 955-61.

19. RiEger N, WATtchow D. The effect of vaginal delivery on anal function. Aust N Z J Surg 1999; 69: 172-7.

20. Fynes M, Donneluy V, Behan M, O’Connell PR, O'Heruhy C. Effect of second vaginal delivery on anorectal physiology and faecal continence: a prospective study. Lancet 1999; 354: 983-6.

21. Eason E, Labrecoue M, Marcoux S, Mondor M. Anal incontinence after childbirth. CMAJ 2002; 166: 32630.

22. Dipiazza D, Richter He, Chapman V, Cliver SP, Neely C, CHEN CC ET AL. Risk factors for anal sphincter tear in multiparas. Obstet Gynecol 2006; 107: 1233-7.

23. Nelson RL, Furner S, Jesudason V. Fecal incontinence in Wisconsin nursing homes. Dis Colon Rectum 1998; 41: 1226-9.

24. Schnelle JF, Macrae PG, Ouslander JG, Simmons SF, NITTA M. Functional Incidental Training, mobility performance, and incontinence care with nursing home residents. J Am Geriatr Soc 1995; 43: 1356-62.

25. Schnelie JF, Alessi CA, Simmons SF, Al-SamarRai NR, BeCK JC, Ouslander JG. Translating clinical research into practice: a randomized controlled trial of exercise and incontinence care with nursing home residents. J Am Geriatr Soc 2002; 50: 1476-83.

26. Topinkova E, Neuwirth J, Stankova M, Melianova A, HAAS T. Urinary and fecal incontinence in geriatric facilities in the Czech Republic. Cas Lek Cesk 1997; 136: 573-7.

27. Aggazzotti G, Pesce F, Grassi D, Fantuzzi G, Righi E, DE VITA D ET AL. Prevalence of urinary incontinence among institutionalized patients: a cross-sectional epidemiologic study in a midsized city in northern Italy. Urology 2000; 56: 245-9. 